\title{
CAMBIO ORGANIZACIONAL Y POLÍTICA DE INCENTIVOS EN LAS UNIVERSIDADES PÚBLICAS: EXPERIENCIA EN EL ÁREA DE LAS CIENCIAS SOCIALES EN UNA UNIVERSIDAD MEXICANA
}

\section{ORGANIZATIONAL CHANGE AND POLITICAL OF INCENTIVES IN THE PUBLIC UNIVERSITIES: EXPERIENCE IN THE AREA OF SOCIAL SCIENCES IN A MEXICAN UNIVERSITY}

\section{RESUMEN}

\author{
Pablo Manuel Chauca Malásquez ${ }^{\mathrm{a}}$
}

El trabajo presenta una interpretación de los factores que posibilitan la aplicación de un sistema de incentivos basado en méritos académicos en las universidades públicas. La discusión se plantea desde la perspectiva del cambio organizacional e institucional. Se argumenta que el desarrollo de un sistema de incentivos en las instituciones públicas de educación superior está asociado a transformaciones en el nivel de los actores académicos (individuos y grupos), en el nivel institucional (reglas y formas de coordinación) y en el nivel de las interacciones con el entorno. Se sostiene que dichas transformaciones implican a su vez cambios en las formas de gobierno, en las formas de gestión, en las fuentes de financiamiento, en las relaciones de enseñanza - investigación, y en los criterios de validación de resultados. Como ilustración, se comenta a lo largo del texto, acerca de la experiencia en el campo de las ciencias sociales en una universidad pública del interior de la república mexicana.

Palabras clave: Cambio organizacional, cambio institucional, sistema de incentivos, formas de trabajo, entorno de aplicación.

\begin{abstract}
The work displays an interpretation of the factors that make possible the application of a system of incentives based on academic merits in the public universities. The discussion considers from the perspective of the organizational and institutional change. It is argued that the development of a system of incentives in the public institutions of superior education is associate to transformations in the level of the academic actors (individual and groups), in the institutional level (rules and forms of coordination) and in the level of the interactions with the surroundings. It is maintained that these transformations imply as well changes in the government forms, in the forms of management, the sources of financing, the relations of education - investigation, and in the criteria of validation of results. Like illustration, one comments throughout the text, about the experience in the field of social sciences in a public university of the interior of the Mexican Republic.
\end{abstract}

Keywords: Organizational change, institutional change, system of incentives, forms of work, surroundings of application.

\section{INTRODUCCIÓN}

El gobierno federal en México, a principios de los años setenta del siglo XX, con la creación del Consejo Nacional de Ciencia y Tecnología (CONACYT) asumió un rol directo en la promoción de la investigación científica y el postgrado. Este rol se intensificó y se reformó en la siguiente década, en la medida en que las políticas gubernamentales para la educación superior e investigación científica adquirieron una nueva legitimidad como parte del proceso de "modernización económica" de corte neoliberal que se inició en los ochenta (1). Una evidencia clara de estos esfuerzos gubernamentales, es que el mayor auge en número de centros de investigación en ciencias sociales y humanidades en México se dio entre 1970 y 1982, teniendo un repunte importante los establecidos fuera de la zona metropolitana de la Ciudad de México, en estados como Veracruz, Sonora, Baja California, México, Sinaloa y Michoacán (2).

En ese contexto, desde los años ochenta y noventa, numerosos establecimientos de educación superior desplegaron estrategias de adaptación a las nuevas exigencias gubernamentales, particularmente a través del CONACYT, y de los programas de Superación Académica

(SUPERA), y para el Mejoramiento del Profesorado (PROMEP). La manera en que las instituciones universitarias mexicanas diseñaron y llevaron a la práctica dichas estrategias de acomodamiento, es el propósito central de este trabajo. En particular, se busca responder dos preguntas principales: ¿cuáles son las estrategias de cambio organizacional e institucional que desplegaron las UPM para elevar los niveles de preparación, calificación y dedicación delos académicos?. Y, ¿cuáles son los impactos y resultados de esas estrategias (en particular del sistema de incentivos y recompensas) en el quehacer docente, de investigación y de vinculación de dichas universidades?.

\section{MATERIAL Y MÉTODOS}

El trabajo se fundamenta en una interpretación del material bibliográfico y documental sobre el tema, en virtud de lo cual se trata de una investigación monográfica. Las reflexiones tienen como referencia básica la experiencia en

${ }^{a}$ Doctor en Ciencias Administrativas. Profesor-investigador titular de la Facultad de Economía de la Universidad Michoacana de San Nicolás de Hidalgo (Morelia, Michoacán, México). Líneas de investigación: Desarrollo de organizaciones, desarrollo empresarial local. 
el área de las ciencias sociales en la Universidad Michoacana de San Nicolás de Hidalgo (UMSNH), ubicada en la ciudad de Morelia, estado de Michoacán, al centro-occidente de México. En esa medida, a lo largo del texto al referirnos a las universidades públicas mexicanas (UPM), en realidad estamos pensando en la citada institución universitaria, que como caso particular no necesariamente refleja la situación del conjunto de las UPM, lo que establece limitaciones al trabajo.

\section{RESULTADOS}

\section{Ideas rectoras}

La investigación se orientó por las siguientes ideas centrales:

a.- Los cambios en el sistema educativo universitario deben entenderse en el marco del cambio social en general $(3,4)$. Esto implica que los cambios educativos tienen interdependencia con las transformaciones en la economía, la política y la organización social, lo que sugiere la necesidad de realizar una investigación interdisciplinar.

b.- El cambio organizacional, se entiende como las transformaciones en las estructuras y funciones reconocidas y aceptadas al interior de una institución universitaria. Y, el cambio institucional, se refiere a las modificaciones en las creencias, normas y reglas formales e informales que permiten el desarrollo de esas funciones y estructuras $(5,6)$.

c.- Toda organización universitaria tiene una relación adaptativa con el entorno y es vista como maleable a las orientaciones de los actores que buscan asegurar sus intereses en entornos inciertos y cambiantes. En ese sentido, es importante tanto estudiar los cambios efectivamente realizados como la manera en que los actores perciben cambios en el entorno, los enfrentan, y dirimen los conflictos que de ello emergen (7).

d.- El cambio en la educación superior no sólo es posible, sino que ocurre todo el tiempo. Pero generalmente es invisible y ocurre incrementalmente. El cambio fundamental es la evolución del conocimiento, materia prima básica de las instituciones públicas de educación superior (IES). Y, la naturaleza de este proceso de cambio en el conocimiento es su progresiva subdivisión y especialización (2).

e.- El desarrollo de un sistema de incentivos y recompensas en las IES está asociado a transformaciones en el nivel de los actores académicos (individuos y grupos), en el nivel institucional (reglas y formas de coordinación) y en el nivel de las interacciones con el entorno. Estas transformaciones implican a su vez cambios en las formas de gobierno, en las formas de gestión, en las fuentes de financiamiento, en las relaciones de enseñanza investigación, y en los criterios de validación de resultados.

\section{Niveles y dimensiones de análisis}

El cambio organizacional e institucional en las UPM debe incorporar tanto los eventos intra-organizacionales como las transformaciones del entorno, y el cambio es un proceso social y político. En ese sentido, conviene distinguir entre cambios "medulares y periféricos" (2). Un ejemplo de cambio medular son los cambios en las formas de gobierno (digamos pasar de una designación de directivos y toma de decisiones que enfatiza la competencia política a una que pone el acento en la competencia académica). En ese nivel, un cambio periférico son las mejoras en procesos administrativos sin afectar clientelas y poderes. Otro ejemplo de cambio medular, son las modificaciones a las bases de la carrera académica y la renovación de planta académica por retiro, rotación y nuevas contrataciones. $Y$, el correspondiente cambio periférico, son los programas de superación de académicos en servicio y/o programas de estímulos sin cambiar la estructura de la carrera académica.

La paradoja en la práctica es que las organizaciones universitarias que efectúan cambios medulares corren el riesgo de generar conflictos que ponen en peligro los propósitos reformadores. Por lo contrario, las universidades que meramente modifican aspectos periféricos conservan la estabilidad; pero posiblemente no alcancen a reformarse significativamente, La experiencia indica que hay que lograr un equilibrio entre modificaciones medulares y periféricas, de tal manera que los cambios sean efectivamente realizados.

Estos tipos de cambio, en las IES se pueden observar en tres niveles de análisis (distinguibles analíticamente, en la realidad están muy interrelacionados): del establecimiento, de la unidad académica y de la disciplina $(2,7)$. En el nivel del establecimiento (IES = UMSNH), los procesos de cambio se refieren a las formas de gobierno y estructura de poder (quiénes deciden sobre asuntos claves, creación de roles y niveles administrativos y políticos, estrategias y tipos de liderazgo); a las formas de gestión (niveles jerárquicos, reglamentación académica y evaluación, política científica interna y política de publicación); y al financiamiento (fuentes internas y externas, formas de asignación de los recursos financieros).

En el nivel de la unidad académica (Facultad de Economía), se consideran los cambios en el modelo académico de las escuelas, facultades e institutos, abarcando dimensiones como: formas de integración de investigación/docencia; formas de trabajo (individuales, grupales, redes de trabajo internas/externas); y capacidad de reproducción del personal académico (formas de reclutamiento, cohortes generacionales, opciones de incorporación de tesistas).

En el nivel de la disciplina, los cambios se dan en: las líneas de trabajo (individuales, prioridades institucionales de largo plazo, agenda flexible en respuesta a demandas externas); los tipos de productos (libros y artículos especializados o de divulgación, de circulación local, nacional e internacional); y las formas y criterios de validación (pares externos, estándares internamente fijados). 


\section{Los cambios realizados}

En el año 2007, la UMSNH está cumpliendo el noventa aniversario de su creación formal, y está regida por la Ley Orgánica promulgada por el Gobierno del Estado de Michoacán en febrero de 1986 (modificada en junio y septiembre de ese mismo año), y por el Estatuto Universitario expedido por el Consejo Universitario en mayo de 1963 (se modificó en 1982, y a la fecha no se ha adecuado a la Ley Orgánica de 1986) (8). La autonomía de la universidad se deposita en la comunidad universitaria y su gobierno está formado por: la comisión de rectoría; el consejo universitario; el rector (dura en su cargo cuatro años y no puede ser reelecto); los consejos técnicos de escuelas, facultades, institutos y unidades profesionales; el consejo de investigación científica; y los directores de escuelas, facultades, institutos y unidades profesionales.

La Comisión de Rectoría (integrada por ocho miembros: cinco son exrectores residentes en la entidad federativa, y los otros tres: decano de los directores de las escuelas, facultades e institutos; decano de los profesores en activo; y consejero universitario estudiante con el más alto promedio de calificaciones en el año lectivo próximo anterior), tiene las atribuciones de: nombrar al rector, conocer de la renuncia de éste y removerlo por causa grave. El Consejo Universitario es la autoridad máxima del gobierno de la universidad, salvo las atribuciones que corresponden a la Comisión de Rectoría, y está integrado por: el rector; los directores de escuelas, facultades, institutos y unidades profesionales; el titular del Consejo de Investigación Científica; un consejero profesor y un consejero alumno propietarios de cada escuela, facultad e instituto; un representante propietario por cada uno de los sindicatos titulares de los contratos colectivos de profesores y trabajadores administrativos; un representante propietario por todas las Casas del Estudiante; y un representante propietario de la Sociedad de Ex alumnos Nicolaítas con derecho a voz únicamente. Por cada representante propietario hay un suplente.

Cada facultad o escuela tiene como autoridad máxima el Consejo Técnico integrado por el director respectivo (elegido por cuatro años por el consejo universitario), un profesor y un alumno por cada grado (elegidos por dos años). La dinámica y funcionamiento académicos de las facultades o escuelas dependen de las decisiones que se adopten en el Consejo Técnico. Cada facultad o escuela formula y pone en práctica su plan de desarrollo institucional acorde con el respectivo plan institucional de toda la universidad.

Por tanto la forma de gobierno y estructura de poder en la UMSNH se basa en una normatividad poco actualizada y con fuentes de autoridad de carácter colegiado. En la práctica predominan más las reglas informales, la centralización de las decisiones claves, y las principales fuentes de autoridad han devenido en burocráticas y personalistas. El proceso de reclutamiento y las promociones del personal académico, están regulados por contrato colectivo (revisado anualmente) y por el reglamento general del personal académico, y en ciertas ocasiones predominan decisiones personales de directivos y de grupos políticos internos. El aparato administrativo está física y funcionalmente disgregado; predominan los procedimientos no codificados y las decisiones casuísticas, y se observa una falta de armonización entre las actividades de docencia, investigación y vinculación con el entorno. Predomina una cultura organizacional tradicional, orientada a administrar recursos existentes en ausencia de visión estratégica desarrollada internamente y de modo participativo. Énfasis en política institucional a nivel licenciatura, y poco a poco se ha ido fortaleciendo una política de evaluación; pero que es usada discrecionalmente en la toma de decisiones y sólo como reacción ante las exigencias gubernamentales a nivel federal.

De ahí que las propias autoridades centrales universitarias han afirmado: "La deficiencia más significativa de nuestra universidad tiene que ver con la baja calidad de los resultados de sus funciones sustantivas... El criterio académico, como principio rector de las tareas y acciones de la Universidad, brilla por su ausencia... Desafortunadamente nuestra Universidad tiene varios años viviendo de sus glorias pasadas, manteniéndolas en la memoria sólo para justificar un presente de pobrezas, rutinas y carencias... Este conservadurismo, este conformismo, resulta la mayor deficiencia de nuestra institución" (9).

En este contexto, la Facultad de Economía (FE) de la UMSNH, ha llevado a cabo desde el año 1998, cambios organizacionales e institucionales de tipo periféricos, es decir, sin modificar las formas de gobierno, la estructura de poder, las formas de gestión, las bases de la carrera académica y las reglas de financiamiento. Se desplegaron programas en siete áreas estratégicas: plan de estudio, personal académico, población estudiantil, investigación, difusión cultural y extensión universitaria, normatividad y organización, e infraestructura y equipamiento. En lo fundamental, estos programas han significado: mejoras en el plan de estudio (flexibilización y actualización); mejoras en los procesos administrativos (mayor formalización); mejoras en la infraestructura (reconstrucción de edificio antiguo, nuevo edificio, mayores acervos bibliográficos); y mejoras en la planta docente $(10,11)$.

En 1997, la planta magisterial de la FE se componía de un total de 28 profesores, de los cuales 22 eran profesoresinvestigadores de tiempo completo (PTC) (8 con grado de maestro). A inicios del 2007, el total del personal académico es de 61 , de los cuales 39 son PTC ( 7 tienen grado de doctor y 23 de maestro) $(11,12)$. Estas mejoras han sido el resultado de: - La superación de académicos en servicio en el marco del programa federal PROMEP. - El reclutamiento de nuevos profesores a través de los programas federales de Retención y Repatriación de Investigadores. - Promociones de profesores dentro de la normatividad universitaria interna. - Programa interno de estímulos que favorece docencia, antigüedad, distribución igualitaria y sin cambiar reglas básicas de asignación. Programa externo de estímulos que favorece la investigación y méritos académicos en el marco del programa federal del Sistema Nacional de Investigadores (SNI). 


\section{DISCUSIÓN}

El estudio de los cambios en el sistema educativo universitario debe realizarse con un enfoque integral e interdisciplinario. El cambio organizacional en las IES es un proceso de múltiples niveles en el que un nivel o sector de la organización es movido por estructuras internas existentes que modelan el cambio emergente, mientras que otro nivel 0 sector se mueve adaptativamente frente a exigencias externas. El entorno institucional, compuesto por las reglas implícitas, los mitos prevalecientes y los rituales del entorno más amplio, son insumos importantes en la explicación del comportamiento y los cambios organizacionales. Interesa distinguir entre cambios ocurridos en la periferia de la organización y en el centro de la misma.

Las UPM han llevado a cabo cambios organizacionales e institucionales en un contexto de una relativa estabilidad política y financiera, y de una más intensa regulación estatal, por lo menos en relación a otras universidades latinoamericanas (2). La Facultad de Economía de la UMSNH, en un marco institucional de continuidad de la forma de gobierno, estructura de poder, forma de gestión y financiamiento, ha realizado cambios organizacionales periféricos e incrementales (pequeños y graduales). Las modificaciones principales se efectuaron a nivel de actores individuales (superación académica personal y alto porcentaje de egresados en la planta magisterial, que fortalecieron la identidad y cultura organizacionales) y grupales (cuerpos académicos y academias que fortalecieron el trabajo en equipo y la articulación entre enseñanza e investigación), que significaron reacomodos y negociaciones sin rupturas y conflictos importantes. Las interacciones con el entorno se debilitaron sobre todo con los grupos de interés del contexto local, aunque se recurrió frecuentemente a las instancias gubernamentales en particular federales para agenciarse de recursos externos.

\section{AGRADECIMIENTOS}

Al Centro de Investigaciones Interdisciplinarias en Ciencias y Humanidades de la Universidad Nacional Autónoma de México, en especial a los doctores Daniel Cazés Menache y Norma Blázquez Graf, por su apoyo para realizar la estancia sabática que nos dio los medios y el tiempo necesarios para que este trabajo fuera posible. Asimismo, a la UMSNH que nos brindó la autorización para el disfrute del año sabático, y la información y la experiencia de vida académica aquí transmitida.

\section{REFERENCIAS BIBLIOGRÁFICAS}

1. Huerta González, Arturo. La política neoliberal de estabilización económica en México: límites y alternativas. pp. 17-62. Editorial Diana. México, 1994.

2. Kent, Rollin; Álvarez, Germán; González, Mario; Ramírez, Rosalba; De Vries, Wietse. Cambio organizacional y disciplinario en unidades de investigación y posgrado en ciencias sociales en México: una visión comparativa. pp. 15-16, 26-29, 45-47, 48-53, 50-55; 70-72. Editores Plaza y Valdés - Centro de Investigación y Estudios Avanzados. México, 2003.

3. Migdal, Joel. Strong Societies and Weak States. State-Society Relations and State Capabilities in the Third World. Princeton University Press. Princeton, USA., 1988.

4. Nee, Víctor; Mathews, Rebecca. Market Transition and Societal Transformation in Reforming State Socialism. Annual Review of Sociology 22. 1996.

5. Appendini, Kirsten; Nuijten, Monique. El papel de las instituciones en contextos locales. Revista de la CEPAL No. 76. Abril, 2002: 21-42. Santiago de Chile.

6. North, Douglass. Instituciones, cambio institucional y desempeño económico. pp. 31-42. Tercera reimpresión de la primera edición en español. Editorial Fondo de Cultura Económica. México, 2006.

7. Scott, W.R. Organizations: Rational, Natural, Open Systems. Englewood Cliffs. Prentice Hall, 1992.

8. Universidad Michoacana de San Nicolás de Hidalgo (UMSNH). Legislación Universitaria I: Normas jurídicas fundamentales. pp. 15-36. Morevallado Editores - UMSNH (Secretaría General). México, 2004.

9. Universidad Michoacana de San Nicolás de Hidalgo (UMSNH). Iniciativa para la reforma universitaria. pp. 30-33. Morevallado Editores - UMSNH (Secretaría General). México, 2003.

10. Facultad de Economía - UMSNH. Plan de Desarrollo Institucional 2004-2010. Morevallado Editores - UMSNH (Facultad de Economía). México, 2005.

11. Escuela de Economía - UMSNH. Plan Institucional de Desarrollo de la Escuela de Economía 2000-2006. Morevallado Editores - UMSNH (Escuela de Economía). México, 2000.

12. 1Facultad de Economía - UMSNH. Información para los Comités Interinstitucionales para la Evaluación de la Educación Superior (CIEES) - Tabla-Guía de Autoevaluación. México, 2007.

E-mail: pchauca@zeus.umich.mx 